\title{
Stress non-uniformity in a hollow cylinder torsional sand specimen
}

\author{
PATRICK J. NAUGHTON \\ Lecturer, School of Engineering, Institute of Technology, Sligo \\ Ballinode, Sligo, Ireland. \\ Tel. +353 719155222, fax.+353 719155390, e-mail: naughton.patrick@itsligo.ie
}

\section{BRENDAN C. O'KELLY}

Lecturer in Civil Engineering

Department of Civil, Structural and Environmental Engineering

Museum Building, Trinity College Dublin, Dublin 2, Ireland.

Tel. +353 16082387, fax.+353 16773072, e-mail: bokelly@tcd.ie

\section{Corresponding author: Brendan O'Kelly}

Published in the International Journal of Geomechanics and Geoengineering, 2007, Vol. 2, No. 2, 117-122.

First submission: 26th September 2006.

Resubmitted: 31st January 2007. 


\begin{abstract}
The hollow cylinder torsional apparatus (HCTA) facilitates more generalized stress path testing although it has been criticized on account of the level of stress nonuniformity that may develop due to the curvature of the test specimen walls. The stress nonuniformity that may develop in dense sand specimens $(35.5-\mathrm{mm}$ inner radius, $50.0-\mathrm{mm}$ outer radius and 200-mm in height) over small-to-medium strain levels in the HCTA were studied using an isotropic linear-elastic stress analysis. The stress nonuniformity, quantified in terms of the stress nonuniformity coefficient $\beta_{R}$, increased in proportion to the stress ratio $R$, and was generally acceptable throughout the stress space for $R \leq 1.5$. Regions where unacceptable stress nonuniformity may develop were identified in the vicinity of (b, $\left.\alpha_{\sigma}\right)=\left(1,0^{\circ}\right),\left(0,90^{\circ}\right)$ and for all values of the $b$ parameter in the vicinity of $\alpha_{\sigma}=45^{\circ}$. Placing restrictions on the difference in the confining pressures that may be applied across the specimen wall thickness was found to reduce the stress nonuniformity but also limited the regions in stress space that could be probed.
\end{abstract}

Keywords: Hollow cylinder torsional apparatus, Sand, Stress path, Stress nonuniformity 


\section{Introduction}

The hollow cylinder torsional apparatus (HCTA) facilitates more generalized stress path testing, including complete control of the rotation of the major principal stress to the vertical direction, and the simulation of complex field loading conditions (Hight et al. 1983). The test specimen, which represents a single point under consideration in the ground, must be subject to a reasonably uniform state of effective stress for accurate interpretations of the experimental data. However, in common with other devices for the shear strength measurement, the HCTA has been criticized (for example Saada and Townsend 1981) on account of the level of stress nonuniformity that may develop. A new HCTA that facilitates stress path testing over a wide strain range has been developed recently by O'Kelly and Naughton $(2003,2005)$ at University College Dublin (UCD) and, in a general context; the study of the stress nonuniformity associated with this apparatus is an important consideration.

Non-zero stresses $\sigma_{\mathrm{z}}, \sigma_{\mathrm{r}}, \sigma_{\theta}$ and $\tau_{\mathrm{z} \theta}$ are induced in the wall thickness of the UCD test specimen $\left(35.5-\mathrm{mm}\right.$ inner radius $r_{i}, 50.0-\mathrm{mm}$ outer radius $r_{0}$, and $200-\mathrm{mm}$ in height $\mathrm{H}$ ) under the system of independently controlled surface loads and confining pressures (figure 1) which simulate the overburden and the system of applied loads. In common with other devices for shear strength measurement, stress nonuniformity develops in the immediate vicinity of the specimen ends (due to frictional restraint and the stiffness of the loading platens) and also due to the wall curvature whenever the stress paths require the application of a torque or differences in the confining pressures that act across the wall thickness (Gens and Potts 1984). Saada et al. (1983) recognized this fact and recommended equalising the inner and outer confining pressures ( $\mathrm{p}_{\mathrm{i}}$ and $\mathrm{p}_{\mathrm{o}}$, respectively) although the intermediate principal stress parameter $\mathrm{b}$ (Eq. (1)) and the orientation of the major principle stress to the vertical direction, $\alpha_{\sigma}$, are then inextricably linked.

$\mathrm{b}=\frac{\sigma_{2}-\sigma_{3}}{\sigma_{1}-\sigma_{3}}$

where $\sigma_{1}, \sigma_{2}$ and $\sigma_{3}$ are the major, intermediate and minor principal stresses.

The level of stress nonuniformity is a function of the specimen size test, decreasing as the wall thickness is reduced or as the inner radius increases (Sayao and Vaid 1991). Previous research using linear-elastic analysis includes that of Vaid et al. (1990) and Sayao and Vaid (1991) for the University of British Colombia (UBC) HCTA $\left(r_{i}=51.0 \mathrm{~mm}, \mathrm{r}_{\mathrm{o}}=76.0 \mathrm{~mm}\right.$, $\mathrm{H}=302 \mathrm{~mm})$ and Hight et al. (1983) for the Imperial College HCTA $\left(\mathrm{r}_{\mathrm{i}}=101.5 \mathrm{~mm}, \mathrm{r}_{\mathrm{o}}=\right.$ $127.0 \mathrm{~mm}, \mathrm{H}=254 \mathrm{~mm}$ ). The stress nonuniformity has also been studied by several researchers using either nonlinear elastic (Wijewickreme and Vaid, 1991) or elasto-plastic models by the Imperial College group (Hight et al. 1983; Gens and Potts, 1984; Menkiti, 1995; Rolo, 2003) which indicated that the level of stress nonuniformity may not be a great as shown by linear elastic type analysis. 
This paper studies the stress nonuniformity that may develop in smaller sand specimens $\left(\mathrm{r}_{\mathrm{i}}\right.$ $=35.5 \mathrm{~mm}, \mathrm{r}_{\mathrm{o}}=50.0 \mathrm{~mm}, \mathrm{H}=200 \mathrm{~mm}$ ) in the UCD HCTA. The inner and outer confining pressures are independently controlled in the UCD apparatus and the effects of frictional end restraint and the stiffness of the loading platens have been reduced to acceptable levels by careful design of the platens, the selection of appropriate specimen dimensions and the measurement of the deformational response over a central 45-mm gauge length (O'Kelly and Naughton 2005).

\section{Quantifying stress nonuniformity}

The shear resistance of sand material is controlled by the friction mobilized at the grain contacts so that it is more convenient to express the principal stress regime in terms of an alternate but equivalent set of stress parameters, namely the mean confining stress, $\mathrm{p}$; the shear stress (or stress ratio, $\mathrm{R}=\sigma_{1} / \sigma_{3}$ ); the intermediate principal stress parameter, $\mathrm{b}$, and the orientation of the major principal stress to the vertical direction, $\alpha_{\sigma}$. The level of stress nonuniformity was quantified in terms of the stress nonuniformity coefficient $\beta_{R}$ (Sayao and Vaid 1991) which relates the maximum difference in the stress ratio $R$, to its mean value acting across the wall thickness:

$\beta_{\mathrm{R}}=\frac{\mathrm{R}_{\text {max }}-\mathrm{R}_{\text {min }}}{\mathrm{R}_{\text {mean }}} \times 100 \quad(\%)$

where $\mathrm{R}_{\max }, \mathrm{R}_{\min }$ and $\mathrm{R}_{\text {mean }}$ are the maximum, minimum and mean values of the major-tominor principal stress ratio.

The stress nonuniformity was considered acceptable for $\beta_{\mathrm{R}}<20 \%$ (as suggested in previous studies, for example Sayao and Vaid (1991)) which corresponded to a $10 \%$ variation from the $\mathrm{R}_{\text {mean }}$ value. The values of the stress components were computed from the applied surface loads $(\mathrm{W}, \mathrm{T})$ and confining pressures $\left(\mathrm{p}_{\mathrm{o}}, \mathrm{p}_{\mathrm{i}}\right)$ by considering the test specimen as a thick-walled hollow cylinder having an isotropic linear-elastic response (Eqs. (3) to (5)). The equations for $\sigma_{z}, \sigma_{\mathrm{r}}$ and $\sigma_{\theta}$ were derived from equilibrium considerations alone. The equation for the circumferential shear stress, $\tau_{\mathrm{z} \theta}$, assumed that a linear distribution of shear stress was mobilized across the wall thickness (Sayao and Vaid 1991). The stress components were averaged over the volume of the UCD specimen. The equations used in the UCD HCTA analysis were similar to those used by Vaid et al. (1990) but differ from those used by Hight et al. (1983) who assumed a uniform stress distribution across the specimen wall thickness and a linear-elastic stress distribution for the radial and circumferential stress components. 


$$
\begin{aligned}
& \sigma_{\mathrm{z}}=\frac{\mathrm{W}}{\pi\left(\mathrm{r}_{\mathrm{o}}{ }^{2}-\mathrm{r}_{\mathrm{i}}{ }^{2}\right)}+\frac{\mathrm{p}_{\mathrm{o}} \mathrm{r}_{\mathrm{o}}{ }^{2}-\mathrm{p}_{\mathrm{i}} \mathrm{r}_{\mathrm{i}}^{2}}{\mathrm{r}_{\mathrm{o}}{ }^{2}-\mathrm{r}_{\mathrm{i}}{ }^{2}} \\
& \sigma_{\mathrm{r}}=\frac{\mathrm{p}_{\mathrm{o}} \mathrm{r}_{\mathrm{o}}{ }^{2}-\mathrm{p}_{\mathrm{i}} \mathrm{r}_{\mathrm{i}}^{2}}{\mathrm{r}_{\mathrm{o}}{ }^{2}-\mathrm{r}_{\mathrm{i}}{ }^{2}} \mp \frac{2 \mathrm{r}_{\mathrm{i}}{ }^{2} \mathrm{r}_{\mathrm{o}}{ }^{2} \boldsymbol{\phi}_{\mathrm{o}}-\mathrm{p}_{\mathrm{i}} \ln \left(\mathrm{r}_{\mathrm{o}} / \mathrm{r}_{\mathrm{i}}\right)}{\mathrm{b}^{2}-\mathrm{r}_{\mathrm{i}}{ }^{2}} \\
& \tau_{\mathrm{z} \theta}=\frac{4 \mathrm{~T}\left(\mathrm{r}_{\mathrm{o}}{ }^{3}-\mathrm{r}_{\mathrm{i}}^{3}\right)}{3 \pi\left(\mathrm{r}_{\mathrm{o}}{ }^{4}-\mathrm{r}_{\mathrm{i}}^{4}\right)\left(\mathrm{r}_{\mathrm{o}}{ }^{2}-\mathrm{r}_{\mathrm{i}}{ }^{2}\right)}
\end{aligned}
$$

The use of a linear-elastic model was considered appropriate in determining the level of stress nonuniformity of dense sands over small-to-medium strain levels, generally associated with serviceability limit state conditions. Furthermore, Wijewickreme and Vaid (1991) showed that both linear-elastic and more realistic hyperbolic constitutive models both identified similar regions in stress space where unacceptable stress nonuniformity may develop.

\section{Stress nonuniformity due to wall curvature}

The values of the stress components that act at intermediate radii in the wall thickness of the UCD specimen were calculated for a series of predefined stress paths in $\mathrm{R}-\mathrm{b}-\alpha_{\sigma}$ space (figure 2). The level of stress nonuniformity was independent of the mean confining stress (set in this study at $\mathrm{p}=500 \mathrm{kPa}$ ) since the axial normal stress acting across the wall thickness was also uniform. While this assumption was not strictly valid next to the ends of the specimen due to end restraint effects, the axial normal stress was uniform within its central 45-mm gauge length. The stress paths originated from the $b-\alpha_{\sigma}$ plane with the start points given by $\mathrm{b}=0,0.25,0.5,0.75$ and 1.0 , and with $\alpha_{\sigma}=0^{\circ}, 22.5^{\circ}, 45^{\circ}, 67.5^{\circ}$ and $90^{\circ}$

(figure 2). The value of R was increased for each stress path while the values of $p, b$ and $\alpha_{\sigma}$ remained fixed. The stress nonuniformity corresponding to R-values of 1.5, 2.0, 2.5, 3.0 and 3.5 were computed. The inner and outer specimen radii remained unchanged from their initial values, which was a reasonable assumption for small-to-medium strains of the dense sand specimens.

The values of the stress components $\left(\sigma_{\mathrm{z}}{ }^{\mathrm{r}},{\sigma_{\mathrm{r}}}_{\mathrm{r}}^{\mathrm{r}},{\sigma_{\theta}}^{\mathrm{r}}, \tau_{\mathrm{z} \theta}{ }^{\mathrm{r}}\right)$ corresponding to a specific radius $\mathrm{r}$ (with $35.5 \leq \mathrm{r} \leq 50.0$ ) were derived from equilibrium considerations assuming an isotropic linear-elastic model (Eqs. (6) to (8), Boresi et al. 1993). The circumferential shear stress was derived assuming a linear shear stress distribution induced across the specimen wall thickness similar to Vaid et al. (1990).

$\sigma_{z}^{r}=\sigma_{z}$ 


$$
\begin{aligned}
& \sigma_{r}^{r}=\frac{p_{o} r_{o}{ }^{2}-p_{i} r_{i}{ }^{r}}{r_{o}{ }^{2}-r_{i}^{2}} \mp \frac{r_{o}{ }^{2} r_{i}^{2} \phi_{o}-p_{i}}{b^{2}-r_{i}{ }^{2} r^{2}} \\
& \sigma_{z \theta}^{r}=\frac{2 T r}{\pi l^{4}-r_{i}^{4}}
\end{aligned}
$$

The values of the stress components were independent of the soil parameters and were functions of the specimen geometry only. The analysis was performed by calculating the stress state at intermediate points along the stress paths shown in figure 2 . The variation in the stress components across the specimen wall thickness at these intermediate points was calculated using Eqs. (6) to (8) from which the corresponding $\beta_{\mathrm{R}}$ stress nonuniformity coefficients were resolved. The analysis examined the stress state, in polar coordinates, of an element of the specimen wall located at mid-height, away from the specimen ends.

\section{Results and discussion}

With the exception of the axial normal stress $\sigma_{z}{ }^{r}$, the computed stress components varied continuously across the wall thickness. For example, a 10-20\% variation occurred about the mean $\sigma_{\mathrm{r}}, \sigma_{\theta}$ and $\tau_{\mathrm{z} \theta}$ values for the particular case of $\mathrm{p}=500 \mathrm{kPa}, \mathrm{R}=3.0, \mathrm{~b}=1$ and $\alpha_{\sigma}$ $=45^{\circ}$ shown in figure 3 . Contour plots of $\beta_{\mathrm{R}}$ versus $\mathrm{b}$ and $\alpha_{\sigma}$ parameters are shown in figure 4 for different values of the stress ratio $\mathrm{R}$. The plots are asymmetric but generally followed a similar pattern with the value of the $\beta_{R}$ coefficient increasing in proportion with the Rvalue. The level of stress nonuniformity was acceptable throughout the stress space for $\mathrm{R} \leq$ 1.5 , with $\beta_{R}<20 \%$. The stress distributions in the vicinity of $\left(b, \alpha_{\sigma}\right)=\left(0,0^{\circ}\right)$ and $\left(1,90^{\circ}\right)$, corresponding to the respective stress states in triaxial compression and triaxial extension, were fully uniform for all R-values studied.

However, significant stress nonuniformity occurred in three regions of stress space; namely, in the vicinity of $\left(b, \alpha_{\sigma}\right)=\left(1,0^{\circ}\right)$ and $\left(0,90^{\circ}\right)$ and in the vicinity of $\alpha_{\sigma}=45^{\circ}$ for all values of the $b$ parameter, figure 4 . The ratio of the inner to outer confining pressures would have been set at its maximum and minimum values for $\left(b, \alpha_{\sigma}\right)=\left(1,0^{\circ}\right)$ and $\left(0,90^{\circ}\right)$, respectively. The applied torque and hence the circumferential shear stress would have been set at its maximum value in the vicinity of $\alpha_{\sigma}=45^{\circ}$. In general, unacceptable stress nonuniformity developed for the following (figure 5):

- $\mathrm{R}>1.5, \mathrm{~b}>0.5$, and $\alpha_{\sigma} \cong 0^{\circ}$,

- $\mathrm{R}>1.5, \mathrm{~b}=0-1$, and $\alpha_{\sigma} \cong 45^{\circ}$,

- $\mathrm{R}>2.25, \mathrm{~b}<0.25$, and $\alpha_{\sigma} \cong 90^{\circ}$. 
In regions of high stress nonuniformity, the $\beta_{R}$ coefficient increased nonlinearly as the stress ratio $R$ increased for all values of the $b$ parameter considered (figure 6 ). The regions where unacceptable stress nonuniformity may develop were similar to those identified for the UBC specimen by Sayao and Vaid (1991). However, the $\beta_{R}$ coefficients for the UCD specimen were considerably lower in the vicinity of $\left(b, \alpha_{\sigma}\right)=\left(0,90^{\circ}\right)$ and considerably higher in the vicinity of $\left(b, \alpha_{\sigma}\right)=(1,0)$ with the discrepancies arising due to the difference in the specimen sizes with the UBC specimen having significantly greater inner and outer radii of 51.0 and $76.0 \mathrm{~mm}$. The level of stress nonuniformity was shown to depend on the inner radius, increasing as the inner radius was reduced.

A scatter plot of $\beta_{R}$ versus the confining pressure ratio corresponding to the stress paths analyzed for the UCD HCTA (figure 7) indicated that there was some merit in limiting the confining pressures as suggested by Hight et al. (1983). The stress nonuniformity was generally acceptable with $\beta_{\mathrm{R}}<20 \%$ for stress paths that restricted the confining pressure ratio within the range $0.9 \leq \mathrm{p}_{\mathrm{o}} / \mathrm{p}_{\mathrm{i}} \leq 1.2$. However, the analysis also indicated that unacceptable stress nonuniformity could develop for $\mathrm{R}>2.0$, including for the condition of equal inner and outer confining pressures. Hence, the philosophy adopted in using the UCD HCTA was not to place restrictions on the ratio of the confining pressures or the regions of generalized stress space that could be probed but rather to mark the stress paths for which unacceptable stress nonuniformity may develop for further analysis.

Menkiti (1995) analysed the Imperial College HCTA using the Imperial College finite element code and a modified Cam Clay model with an associated flow rule. In general, the results confirmed that a large specimen $(50.0-\mathrm{mm}$ inner radius and $25.0 \mathrm{~mm}$ in wall thickness) would not have significant stress nonuniformity. Rolo (2003) using finite element analysis of a specimen similar in size to the UCD HCTA showed that under plane strain conditions $\left(\alpha_{\sigma}=0^{\circ}\right)$ where the greatest difference occurs between the inner and outer confining pressures generated the largest stress nonuniformity. The level of stress nonuniformity reduced as the $\mathrm{b}$ parameter was reduced, which is consistent with the results from this study. However, Rolo (2003) did not find a significant variation in the stress nonuniformity in the vicinity of $\alpha_{\sigma}=45^{\circ}$ although this could be a consequence of this study only examining the stress state at the specimen mid-height only.

\section{Summary and conclusions}

Stress nonuniformity develops across the specimen wall thickness in the HCTA due to the wall curvature and, in the immediate vicinity of the specimen ends, due to frictional restrain and the stiffness of the loading platens. The level of stress nonuniformity that may develop in sand specimens (35.5-mm inner radius, 50.0-mm outer radius and 200-mm in height) were studied using an isotropic linear-elastic analysis. With the exception of the axial normal stress, the stress components within the central gage length were found to vary continuously across the wall thickness. The level of stress nonuniformity, quantified in 
terms of the stress nonuniformity coefficient, was found to be acceptable for $\mathrm{R} \leq 1.5$ throughout the stress space, with $\beta_{R}<20 \%$.

Regions corresponding to locations where the applied torque or the difference in the confining pressures were greatest were identified where unacceptable stress nonuniformity may develop; namely, in the vicinity of $\left(b, \alpha_{\sigma}\right)=\left(1,0^{\circ}\right),\left(0,90^{\circ}\right)$ and in the vicinity of $\alpha_{\sigma}=$ $45^{\circ}$ for all values of the $b$ parameter,. The same regions were identified by Sayao and Vaid (1991) although the $\beta_{\mathrm{R}}$ values computer for the UCD HCTA specimen were different on account of the different specimen sizes.

In general, acceptable levels of stress nonuniformity were found to develop when the ratio of the confining pressures was limited within the range $0.9 \leq \mathrm{p}_{\mathrm{o}} / \mathrm{p}_{\mathrm{i}} \leq 1.2$, although this restricted the extent of stress space that could be probed. Little advantage was gained in equalising the inner and outer confining pressures as recommended by Saada et al. (1983), with the analysis indicating that unacceptable stress nonuniformity could develop in any event for $\mathrm{R}>2.0$. 


\section{References}

Boresi, A.P., Schmidt, R.J. and Sidebottom, O.M., Advanced mechanics of materials, 1993 (John Wiley \& Sons: New York).

Gens, A. and Potts, D.M., Formulation of quasi-axisymmetric boundary value problems for finite element analysis. Engineering Computations, 1984, 1(2), 144-150.

Hight, D.W., Gens, A. and Symes, M.J., The development of a new hollow cylinder apparatus for investigating the effects of principal stress rotation in soils. Geotechnique, 1983, 33(4), 355-383.

Menkiti, C.O., Behaviour of clay and clayey-sand, with particular reference to principal stress rotation. PhD thesis, Imperial College of Science, Technology and Medicine, University of London, 1995.

O'Kelly, B.C. and Naughton, P.J., Development of the University College Dublin hollow cylinder apparatus, in 56th Annual Canadian Geotechnical Conference, 2003, Winnipeg, Ontario, CD Rom.

O'Kelly, B.C. and Naughton, P.J., Development of a new hollow cylinder apparatus for stress path measurements over a wide strain range. ASTM Geotechnical Testing Journal, 2005, 28(4), 345-354.

Rolo, R., The anisotropic stress-strain-strength behaviour of brittle sediments. $P h D$ thesis, Imperial College of Science, Technology and Medicine, University of London, 2003.

Saada, A.S., Fries, G. and Ker, C-C., An evaluation of laboratory testing techniques in soil mechanics. Soils and Foundations, 1983, 23(2), 98-112.

Saada, A.S. and Townsend, F.C., State of the art: laboratory strength testing of soils. In Laboratory shear strength of soils (STP 740), pp. 7-77, 1981 (American Society for Testing and Materials: Philadelphia).

Sayao, A. and Vaid, Y.P., A critical assessment of stress nonuniformities in hollow 
cylinder test specimens. Soils and Foundations, 1991, 31(1), 60-72.

Vaid, Y., Sayão, A., Hou, E. and Negussey, D., Generalised stress-path dependent behaviour with a new hollow cylinder torsional apparatus. Canadian Geotechnical Journal,1990 27, pp. 601-616.

Wijewickreme, D. and Vaid, Y.P., Stress nonuniformities in hollow cylinder torsional specimens. ASTM Geotechnical Testing Journal, 1991, 14(4), 349-362. 


\section{List of figure captions:}

Figure 1. Stress state in HCTA specimen.

Figure 2. Stress paths analyzed for the UCD specimen.

Figure 3. Stress distribution across wall thickness $\left(p=500 \mathrm{kPa}, \mathrm{R}=3.0, \mathrm{~b}=1.0, \alpha_{\sigma}=45^{\circ}\right)$.

Figure 4. Values of $\beta_{R}$ coefficient in $b-\alpha_{\sigma}$ space for $R=1.5-3.5$.

Figure 5. Areas where unacceptable stress non-uniformity may arise.

Figure 6(a) $\alpha_{\sigma}=0^{\circ}$.

Figure $6(\mathrm{~b}) \alpha_{\sigma}=45^{\circ}$.

Figure 6(c) $\alpha_{\sigma}=90^{\circ}$.

Figure 6. Variation of $\beta_{R}$ with increasing stress ratio, $R$.

Figure 7. Variation of $\beta_{R}$ with confining pressure ratio, $p_{o} / p_{i}$.
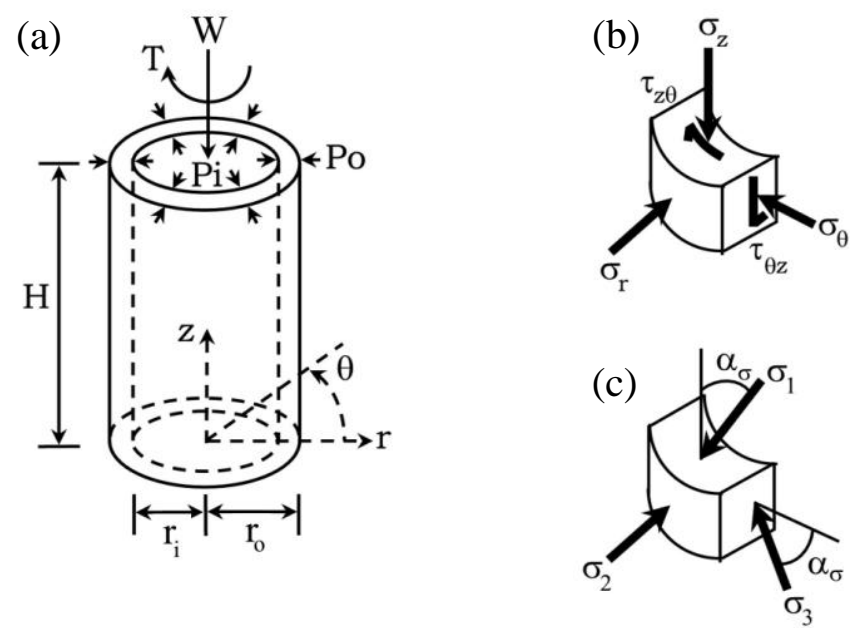

Fig. 1. Stress state in HCTA specimen. 


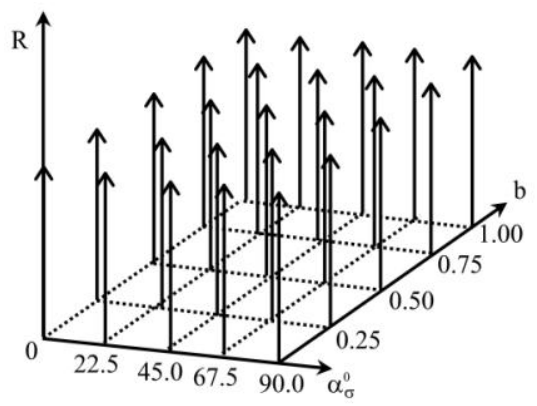

Fig. 2. Stress paths studied for the UCD specimen.

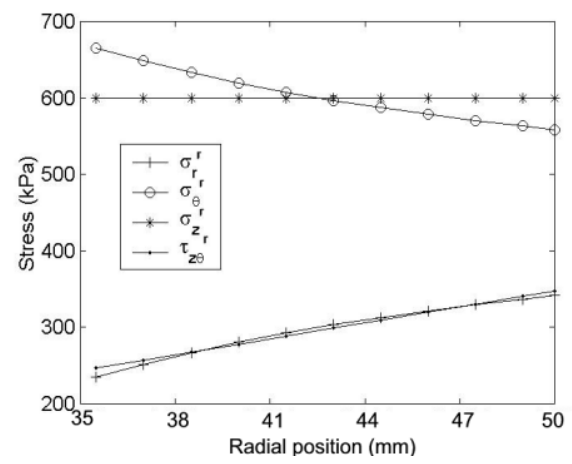

Fig. 3. Stress distribution across wall thickness ( $\left.p=500 \mathrm{kPa}, \mathrm{R}=3.0, \mathrm{~b}=1.0, \alpha_{\sigma}=45^{\circ}\right)$. 

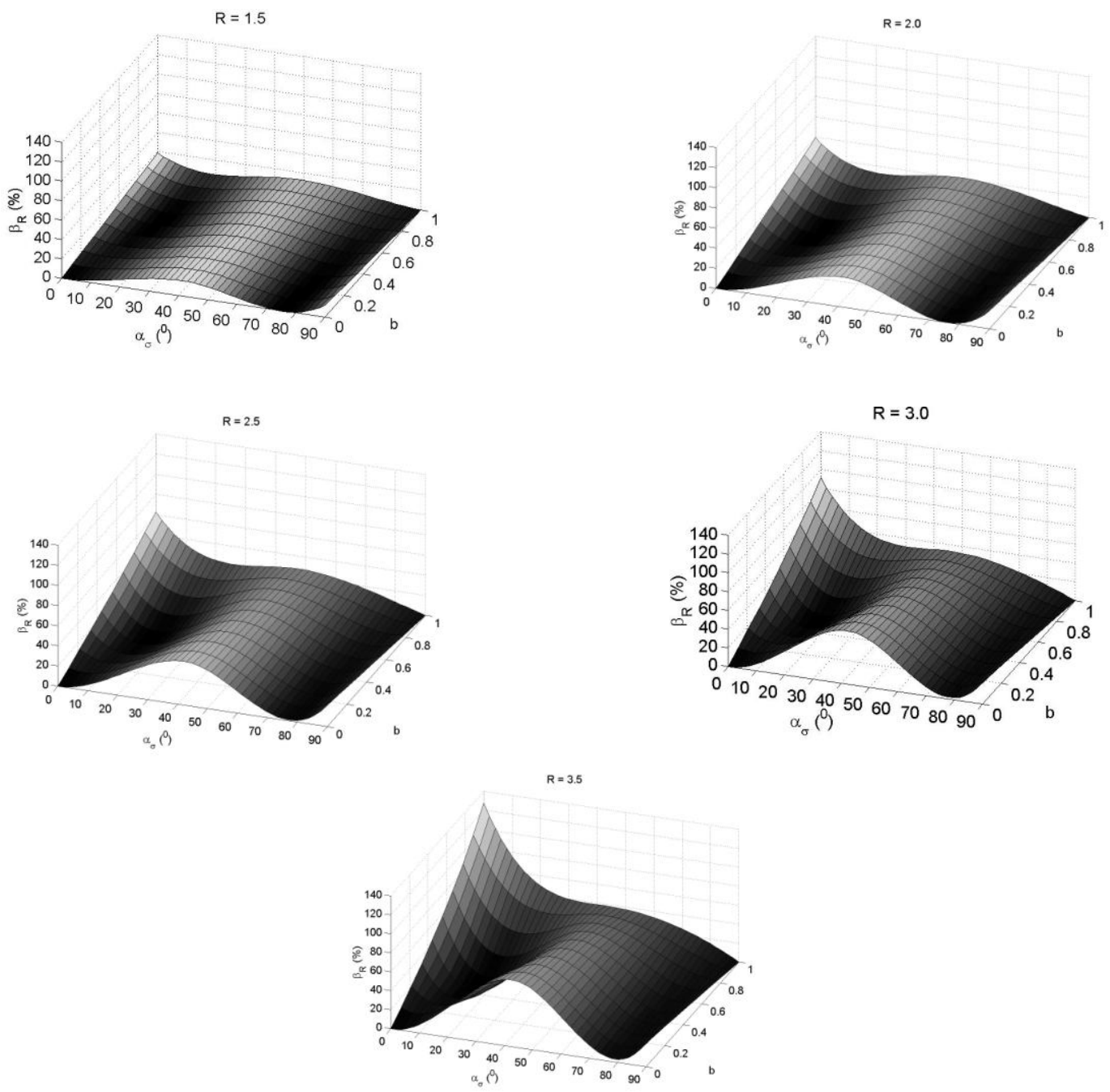

Fig. 4. Values of $\beta_{R}$ coefficient in $b-\alpha_{\sigma}$ space for $R=1.5-3.5$. 


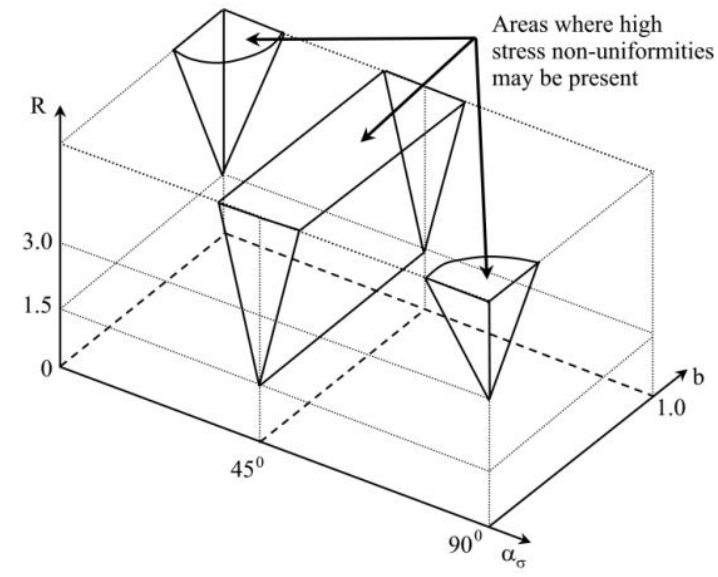

Fig. 5. Areas where unacceptable stress non-uniformity may arise. 
(a)

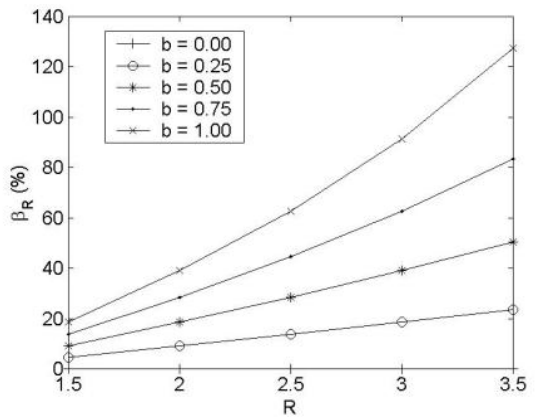

Figure 6 (a) $\alpha_{\sigma}=0^{\circ}$.

(b)

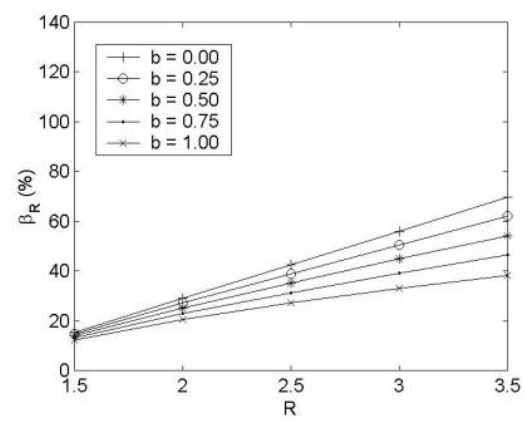

Figure $6(b) \alpha_{\sigma}=45^{\circ}$.

(c)

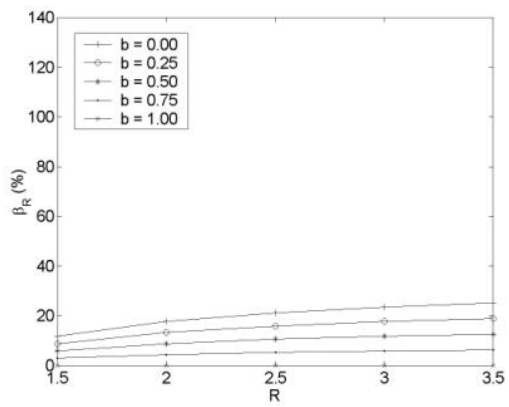

Figure $6(\mathrm{c}) \alpha_{\sigma}=90^{\circ}$.

Fig. 6. Variation of $\beta_{R}$ with increasing stress ratio, $R$. 


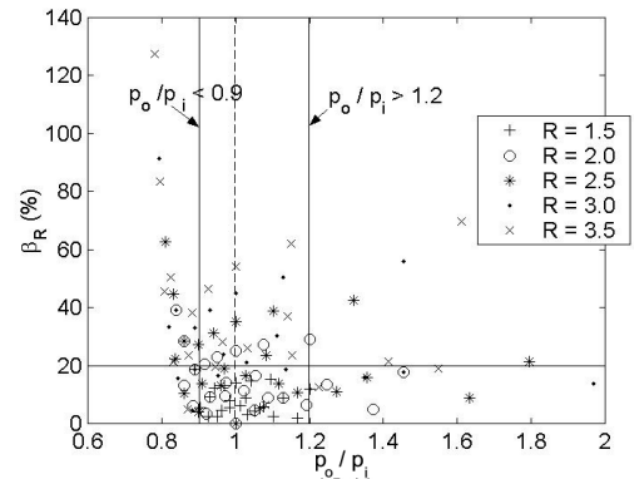

Fig. 7. Variation of $\beta_{R}$ with confining pressure ratio, $p_{o} / p_{i}$. 